\title{
Radiografía de tórax de control en pacientes con neumotórax postraumático asintomático
}

\author{
Follow-up chest x-ray in patients with asymptomatic posttraumatic \\ pneumothorax
}

\author{
Julieta Correa-Restrepo', Mónica Restrepo-Moreno1, Luis Guillermo Peláez², \\ Rafael Díaz-Cadavid², Yuliana López-Vasco², María Alejandra Rojas ${ }^{3}$, \\ David Alejandro Mejía-Toro ${ }^{4}$, Carlos Hernando Morales-Uribe ${ }^{5}$
}

\begin{abstract}
Médico, residente de Cirugía General, Universidad de Antioquia, Medellín, Colombia
Estudiante de Medicina, Universidad de Antioquia, Medellín, Colombia

Médica general, Universidad de Antioquia, Medellín, Colombia

Médico, cirujano general, especialista en Cirugía de Trauma y Emergencias; profesor, Cirugía General, Universidad de Antioquia; cirujano asistencial, Hospital Universitario San Vicente Fundación, Medellín, Colombia

5 Médico, cirujano general, M.Sc. Epidemiología Clínica; profesor titular de Cirugía General, Universidad de Antioquia; cirujano asistencial, Hospital Universitario San Vicente Fundación, Medellín, Colombia
\end{abstract}

\section{Resumen}

Introducción. El trauma de tórax tiene alta incidencia y el neumotórax es el hallazgo más frecuente. La literatura es escasa sobre qué hacer con los pacientes asintomáticos y con neumotórax por trauma de tórax penetrante.

El objetivo de este estudio fue evaluar cuáles son los hallazgos de la radiografía de control de los pacientes con trauma de tórax penetrante que no son llevados inicialmente a cirugía, y su utilidad para determinar la necesidad de un tratamiento adicional.

Métodos. Se realizó un estudio retrospectivo de cohorte, incluyendo pacientes mayores de I5 años que ingresaron por trauma de tórax penetrante entre enero de 2015 y diciembre de 2017 y que no requirieron manejo quirúrgico inicial. Se analizaron los resultados de la radiografía de tórax, el tiempo de su toma y la conducta decidida según los hallazgos en los pacientes dejados inicialmente bajo observación.

Resultados. Se incluyeron I.554 pacientes, cuya edad promedio fue de 30 años, 92,5\% del sexo masculino y $97 \%$ con herida por arma cortopunzante. Se dejaron 36I pacientes bajo observación con radiografía de control, de los cuales I86 (5I,5\%) no presentaban alteraciones en su radiografía inicial, I42 tenían neumotórax menor del 30 \%

Fecha de recibido: 30/06/2019 - Fecha aceptación: 21/08/2019

Correspondencia: Julieta Correa-Restrepo, Calle 40\#101A-165, Apartamento 333, Medellín, Colombia Teléfono: (317) 895-7774

Correo electrónico: julicore2003@gmail.com

Citar como: Correa-Restrepo J, Restrepo-Moreno R, Peláez LG, Díaz-Cadavid R, López-Vasco Y, Rojas MA, Mejía-Toro DA, MoralesUribe $\mathrm{CH}$. Radiografía de tórax de control en pacientes con neumotórax postraumático asintomático. Rev Colomb Cir. 2020;35:75-83. https://doi.org/10.30944/20117582.590

Este es un artículo de acceso abierto bajo una Licencia Creative Commons - BY-NC-ND https://creativecommons.org/licenses/by-ncnd/4.0/deed.es 
y 33 tenían neumotórax mayor del $30 \%$, hemoneumotórax o hemotórax. Se requirió toracostomía cerrada como conducta final en 78 casos, esternotomía o toracotomía en 2 casos y 28I se dieron de alta.

Conclusión. En pacientes asintomáticos con neumotórax pequeño o moderado y sin otras lesiones significativas, podrían ser innecesarios los largos tiempos de observación, las radiografías y la toracostomía cerrada.

Palabras clave: traumatismos torácicos; neumotórax; diagnóstico; diagnóstico por imagen; radiografía torácica; tratamiento conservador.

\begin{abstract}
Introduction: Chest trauma has a high incidence, and pneumothorax is the most frequent finding. The literature is limited about what to do with asymptomatic patients with pneumothorax due to penetrating chest trauma. The objective of this study was to evaluate the findings of control follow-up chest $\mathrm{x}$-ray in patients with penetrating chest trauma who are not initially taken to surgery and its usefulness in determining the need for additional management.
\end{abstract}

Methods: A retrospective cohort study was conducted, including patients older than 55 years who were admitted for penetrating chest trauma between January 2015 and December 2017 and who did not require initial surgical management. The results of the chest $x$-ray, the timing of it and the management according to the findings in the patients initially left under observation were analyzed.

Results: I,554 patients were included; whose average age was 30 years, $92.5 \%$ were males and $97 \%$ sustained a gunshot wound; 36I patients were left under observation with control x-ray, of which I86 (5I,5\%) had no findings on their initial radiograph, I42 had pneumothorax less than $30 \%$ and 33 had pneumothorax greater than $30 \%$, hemoneumotorax or hemothorax. Closed thoracostomy was required as final management in 78 cases, sternotomy or thoracotomy in 2 cases and discharge in 28I.

Conclusion: In asymptomatic patients with small or moderate pneumothorax and without other significant injuries, longer observation times, $\mathrm{x}$-rays and closed thoracostomy may be unnecessary.

Keywords: thoracic injuries; pneumothorax; diagnosis; diagnostic imaging; radiography, thoracic; conservative treatment.

\section{Introducción}

El trauma de tórax se presenta con frecuencia en el mundo y es más común en los países en desarrollo; corresponde al $60 \%$ de los politraumatismos, con una morbilidad y mortalidad hasta del $25 \%$.

El neumotórax traumático es el hallazgo más frecuente y consiste en la acumulación de aire entre la pleura parietal y la visceral. Su diagnóstico se hace mediante radiografía postero-anterior de tórax en posición vertical y, con menor sensibilidad, en posición supina (79 Vs. $8 \%)^{2}$.

El neumotórax se produce por una lesión directa o indirecta sobre el tórax, que genera un mecanismo de válvula con sello alrededor de la herida en el plano subcutáneo, lo cual permite la entrada de aire al espacio pleural. Sin embargo, la fisiopatología del neumotórax tardío o los hallazgos tardíos cambian, porque se deben a trombos y adherencias que se desintegran con el tiempo, permitiendo el paso de aire de forma tardía ${ }^{3,4}$. Las localizaciones anatómicas más frecuentes son la anterior, la basal y la apical ${ }^{5}$.

La presentación clínica varía ampliamente y es en los servicios de urgencias de alta demanda donde los pacientes asintomáticos se convierten en un factor distractor, generando la necesidad de periodos de observación y estudios adiciona- 
les, pues se considera que el $2 \%$ puede desarrollar neumotórax de manera tardía, para lo cual se necesitan radiografías de control y examen físico seriado $^{6}$. La radiografía de control se toma usualmente en las seis horas siguientes al trauma, pero en algunos estudios, se ha demostrado que sería suficiente menos tiempo de seguimiento, sin riesgos significativos ${ }^{7,8}$.

Este estudio se desarrolló ante la escasa literatura sobre la conducta que se debe seguir en los pacientes asintomáticos o con hallazgos leves en las imágenes de tórax iniciales, el desconocimiento de los datos epidemiológicos y el número de lesiones diagnosticadas con las radiografías de control, además de la falta de información sobre la incidencia local de lesiones intratorácicas que se pueden manifestar tardíamente y su asociación con otras complicaciones.

Su propósito fue evaluar cuáles eran los hallazgos de la radiografía de control en los pacientes con trauma de tórax penetrante que no son llevados inicialmente a cirugía y su utilidad para determinar la necesidad de un manejo adicional.

\section{Métodos}

Se realizó un estudio retrospectivo de cohorte con pacientes mayores de I5 años que ingresaron al Hospital Universitario San Vicente Fundación de Medellín, entre enero de 2015 y diciembre de 2017 .

Los criterios de inclusión fueron diagnóstico de trauma penetrante de tórax y que no hubieran requerido tratamiento quirúrgico inicial. Se excluyeron los pacientes con inestabilidad hemodinámica o que hubieran sido sometidos a cirugía urgente, que solicitaron el egreso voluntario y que hubieran recibido atención extrainstitucional. Para la búsqueda, se incluyeron los códigos de la Clasificación Internacional de Enfermedades en su décima edición (CIE-Io) relacionados con trauma de tórax.

Se revisaron las historias clínicas y se tomaron los datos demográficos de edad y sexo, y los datos clínicos como tipo de herida y localización, signos vitales al ingreso, tiempo entre el trauma y la radiografía inicial, hallazgos en la radiogra- fía y otros estudios solicitados, conducta inicial, tiempo entre la radiografía inicial y la de control, hallazgos en la radiografía control y conducta final, al igual que la necesidad de reingreso por la misma causa y diagnóstico definitivo.

Se evaluó como resultado principal el cambio entre la radiografía inicial y la de control, con la necesidad de intervención quirúrgica en los pacientes dejados bajo observación inicialmente.

Los datos recolectados se registraron en una base de datos de Excel $^{\mathrm{TM}}$ de Microsoft Office 2013. Con el software Stata ${ }^{\mathrm{TM}}$, versión 22, se hizo un análisis descriptivo de las variables, las tablas y los gráficos de los resultados obtenidos. Para las variables continuas, se utilizaron medidas de resumen y, para las variables cualitativas, frecuencias absolutas y relativas.

\section{Resultados}

Se analizaron 3.543 historias clínicas. Se excluyeron I.989 por las siguientes causas: I.I5I pacientes presentaban trauma cerrado, I4I firmaron el alta voluntaria antes de la toma de la radiografía de tórax, 246 requirieron intervención quirúrgica emergente sin examen previo de imágenes, 2I2 correspondían a revisiones, y Io fallecieron al momento del ingreso. Las demás causas de exclusión se listan en la figura I. Al final, se incluyeron I.554 pacientes.

El promedio de edad fue de 30 años, la mediana de 27 años, con un mínimo de 15 años y un máximo de 93 años (rango intercuartílico de 21 a 36 años). El 92,5\% de los pacientes afectados fueron de sexo masculino. Durante la evaluación inicial, el promedio de la presión arterial sistólica fue 120 $\mathrm{mm} \mathrm{Hg}$ y, el de la frecuencia cardiaca, 90 latidos por minuto, con un rango de 79 a I03 (tabla I).

La localización más frecuente de la herida fue en la zona posterior del tórax $(44,6 \%)$ seguida por la anterior (30,8\%), y el I3,8 \% de los pacientes presentó heridas múltiples. En el $97 \%$ de los pacientes, el objeto causal fue un arma cortopunzante; en los restantes, la causa fue una herida por proyectil de arma de fuego.

A la mitad de los pacientes se les tomó una radiografía de tórax durante la primera hora 


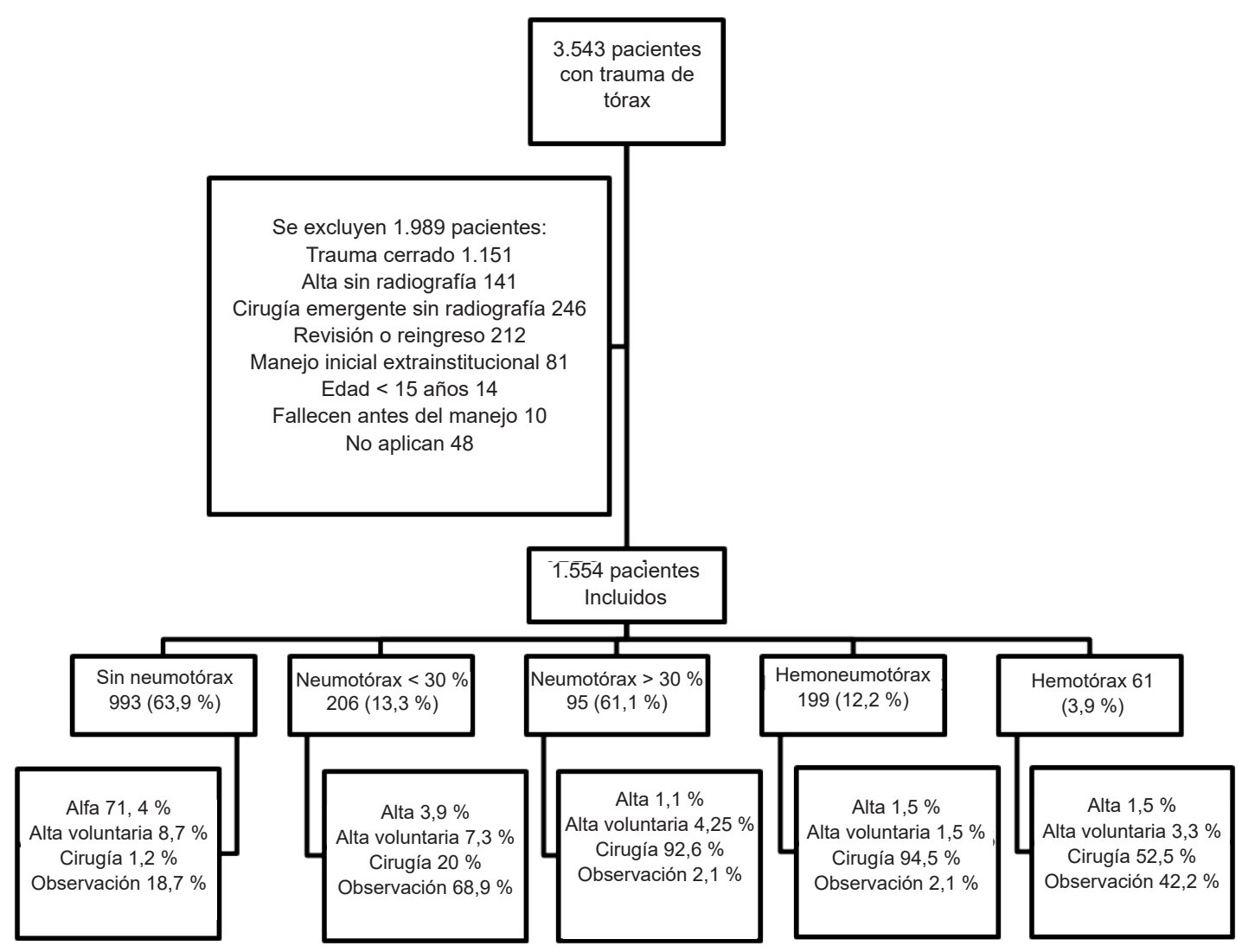

Figura 1. Pacientes incluidos, criterios de exclusión y resultado de la radiografía inicial.

Tabla 1. Características demográficas y clínicas de los 1.554 pacientes incluidos

\begin{tabular}{|c|c|}
\hline Variable & $\begin{array}{c}n(\%) \\
\text { o mediana (RIC) }\end{array}$ \\
\hline Edad & $27(21-36)$ \\
\hline Sexo masculino & $1.437(92,5)$ \\
\hline Frecuencia cardiaca (latidos por minuto) & $90(79-103)$ \\
\hline Presión arterial sistólica $(\mathrm{mm} \mathrm{Hg})$ & $120(109-130)$ \\
\hline Presión arterial diastólica $(\mathrm{mm} \mathrm{Hg})$ & $76(67-80)$ \\
\hline Frecuencia respiratoria & $18(17-20)$ \\
\hline \multicolumn{2}{|l|}{ Localización de la herida } \\
\hline Tórax, posterior & $693(44,6)$ \\
\hline Tórax, anterior & $479(30,8)$ \\
\hline Toracoabdominal izquierda & $79(5,1)$ \\
\hline Toracoabdominal derecha & $65(4,2)$ \\
\hline Zona 1 de cuello & $24(1,5)$ \\
\hline Heridas múltiples & $214(13,8)$ \\
\hline \multicolumn{2}{|l|}{ Tipo de herida } \\
\hline Arma cortopunzante & $1.508(97)$ \\
\hline Proyectil de arma de fuego & $46(3)$ \\
\hline
\end{tabular}

después del trauma y, a un tercio, entre una y tres horas después. En esta primera radiografía, $993(63,9 \%)$ pacientes no presentaron hallazgos anormales, 206 (I3,3\%) presentaron neumotórax menor del $30 \%$. El 27,3\% requirieron una ecografía pericárdica como estudio adicional, principalmente, aquellos con una herida anterior, con el fin de descartar lesiones cardiacas que requirieran intervención quirúrgica urgente; el resto no necesitó ninguna ayuda diagnóstica adicional.

Con la radiografía inicial, se estableció la necesidad de toracostomía en $\mathbf{2 2} \%$ de los casos, de toracotomía o esternotomía, en I,3 \%, y de observación, en 23,2\%; además, se decidió el alta en el 46,5\% (tabla 2). De los 993 pacientes con radiografía inicial sin hemotórax o neumotórax, a $709(7 \mathrm{I}, 4 \%)$ se les dio de alta sin estudios adicionales; I86 (I8,7\%) se dejaron bajo observación y a 86 
(8,7\%) se les indicó observación clínica y radiografia de control, pero se negaron al tratamiento y se retiraron voluntariamente del servicio.

Se dejaron bajo observación 36I pacientes, I86 $(79,6 \%)$ sin neumotórax y I42 (IO,2 \%) con neumotórax menor del 30\%, 2 (I,I \%) con neumotórax mayor del 30\%, 5 (5,4 \%) con hemoneumotórax y $26(3,8 \%)$ con hemotórax menor de $300 \mathrm{ml}$. Se les tomó una radiografía de control a $9(2,5 \%)$ pacientes durante las primeras tres horas después del trauma, a 202 (56\%), entre tres y seis horas, y a I50 (4I,6\%), después de seis horas.
De los I86 pacientes con una radiografía inicial sin ningún hallazgo, I48 (79,6\%) continuaron sin cambios y I6I $(86,6 \%)$ fueron dados de alta, mientras que I9 (I0,2\%) presentaron un neumotórax menor del $30 \%$ y I9(I0 \%) presentó neumotórax mayor del $30 \%$, hemoneumotórax o hemotórax, requiriéndose en 25 ( $13,4 \%)$ casos toracostomía por el hallazgo en el control o por presentar síntomas (disnea, desaturación) (tablas 3 y 4 ).

En los dos pacientes con neumotórax mayor del $30 \%$ el hallazgo en la radiografía de con-

Tabla 2. Conducta tomada con la radiografía de tórax del ingreso

\begin{tabular}{|c|c|c|c|c|c|c|}
\hline \multirow[b]{2}{*}{ Conducta } & \multicolumn{5}{|c|}{ Resultado de radiografía inicial } & \multirow[b]{2}{*}{ Total } \\
\hline & $\begin{array}{c}\text { Sin neumotórax: } \\
993(63,9 \%)\end{array}$ & $\begin{array}{c}\text { Neumotórax }<30 \% \text { : } \\
206(13,3 \%)\end{array}$ & $\begin{array}{c}\text { Neumotórax }>30 \% \text { : } \\
95(6,1 \%)\end{array}$ & $\begin{array}{l}\text { Hemoneumotórax: } \\
199(12,8 \%)\end{array}$ & $\begin{array}{l}\text { Hemotórax: } \\
61(3,9 \%)\end{array}$ & \\
\hline Toracotomía & $3(0,3)$ & $3(1,5)$ & - & $7(3,5)$ & $2(3,3)$ & $15(1,0)$ \\
\hline $\begin{array}{l}\text { Toracostomía } \\
\text { cerrada }\end{array}$ & $7(0,7)$ & $38(18,5)$ & $88(92,6)$ & $179(90)$ & $30(49,2)$ & $342(22,0)$ \\
\hline Esternotomía & $2(0,2)$ & - & - & $2(1)$ & - & $4(0,3)$ \\
\hline Observación & $186(18,7)$ & $142(68,9)$ & $2(2,1)$ & $5(2,5)$ & $26(42,2)$ & $361(23,2)$ \\
\hline Alta & $709(71,4)$ & $8(3,9)$ & $1(1,1)$ & $3(1,5)$ & $1(1,6)$ & $722(46,5)$ \\
\hline Alta voluntaria & $86(8,7)$ & $15(7,3)$ & $4(4,25)$ & $4(1,5)$ & $2(3,3)$ & $110(7,1)$ \\
\hline
\end{tabular}

Tabla 3. Resultados de la radiografía de control en el grupo de pacientes bajo observación $(n=361)$

\begin{tabular}{lccccc}
\hline Variable & $\begin{array}{c}\text { Sin neumotórax } \\
\mathbf{1 8 6}(\mathbf{7 9 , 6} \%)\end{array}$ & $\begin{array}{c}\text { Neumotórax }<30 \% \\
\mathbf{1 4 2}(\mathbf{1 0 , 2} \%)\end{array}$ & $\begin{array}{c}\text { Neumotórax }>\mathbf{3 0} \% \\
\mathbf{2}(\mathbf{1 , 1} \mathbf{\%})\end{array}$ & $\begin{array}{c}\text { Hemoneumotórax } \\
\mathbf{5}(\mathbf{5 , 4} \mathbf{\%})\end{array}$ & $\begin{array}{c}\text { Hemotórax } \\
\mathbf{2 6}(\mathbf{3}, \mathbf{8} \%)\end{array}$ \\
\hline Sin neumotórax & $148(79,6)$ & $22(15,5)$ & - & - & $6(23,1)$ \\
Neumotórax $<30 \%$ & $19(10,2)$ & $81(57)$ & - & $1(20)$ & $1(3,9)$ \\
Neumotórax $>30 \%$ & $2(1,1)$ & $15(10,6)$ & $2(100 \%)$ & - & - \\
Hemoneumotórax & $10(5,4)$ & - & - & $4(80)$ & $5(19,2)$ \\
Hemotórax & $7(3,8)$ & $24(16,9)$ & - & - & $14(53,9)$ \\
\hline
\end{tabular}

Tabla 4. Conducta después de la radiografía de control en los pacientes bajo observación

\begin{tabular}{|c|c|c|c|c|c|}
\hline Variable & $\begin{array}{c}\text { Sin neumotórax } \\
186(79,6 \%)\end{array}$ & $\begin{array}{c}\text { Neumotórax }<30 \% \\
142(10,2 \%)\end{array}$ & $\begin{array}{c}\text { Neumotórax }>30 \% \\
2(1,1 \%)\end{array}$ & $\begin{array}{c}\text { Hemoneumotórax } \\
5(5,4 \%)\end{array}$ & $\begin{array}{l}\text { Hemotórax } \\
26(3,8 \%)\end{array}$ \\
\hline Toracotomía & - & $1(0,7)$ & - & - & - \\
\hline Toracostomía cerrada & $25(13)$ & $42(29,6)$ & $2(100)$ & $1(20)$ & $8(30,8)$ \\
\hline Esternotomía & - & - & - & - & $1(3,9)$ \\
\hline Alta & $161(86,6)$ & $99(69,7)$ & - & $4(80)$ & $17(65,4)$ \\
\hline
\end{tabular}


trol persistió y fueron llevados a toracostomía cerrada. De los cinco pacientes con hemoneumotórax, uno presentó mejoría radiológica sin intervenciones, a uno se le practicó toracostomía y cuatro fueron dados de alta. De 26 pacientes con hemotórax dejados bajo observación, ocho requirieron toracostomía; uno, esternotomía, y I7 fueron dados de alta.

Reingresaron I9 (I,2 \%) pacientes por causas relacionadas con el trauma y, de estos, en io se documentó neumotórax, en 6, hemotórax, y en 3, empiema, con necesidad de intervención en 7 . El resto se dio de alta.

\section{Discusión}

El neumotórax traumático es una lesión común, tanto en trauma cerrado como penetrante de tórax. Puede llegar a generar un riesgo para la vida, con necesidad de intervenciones quirúrgicas urgentes, principalmente en pacientes sintomáticos con hallazgos pleurales, lesiones asociadas o necesidad de asistencia respiratoria mecánica. No obstante, en los pacientes asintomáticos, el tratamiento y el tiempo de observación han sido controversiales.

No se ha establecido un método para determinar el tamaño exacto de un neumotórax en una radiografía. Para esto se han utilizado diferentes estrategias, como el índice de Light, el método de Rhea y el de Collins. El índice de Light consiste en medir el diámetro del pulmón colapsado y el diámetro del hemitórax, asumiendo que la razón entre el cubo de ambas medidas se correlaciona con el volumen del neumotórax ${ }^{9}$. En el método de Collins, al igual que en el de Rhea, se miden tres distancias interpleurales, pero se relacionan con las medidas tomadas con la tomografía computarizada (TC) helicoidal ${ }^{\text {1o }}$.

Además de estos tres métodos clásicos para medir el neumotórax, existen guías que recomiendan otros parámetros para calcular el tamaño del neumotórax. En el 200I, el American College of Chest Physicians (ACCP) clasificó los neumotórax como pequeños o grandes según la distancia interpleural en el ápice pulmonar: menor o mayor de $3 \mathrm{~cm}$, respectivamente ${ }^{\text {II }}$. En el
2003, la British Thoracic Society (BTS) los clasificó de igual forma, pero según una distancia interpleural a la altura de los hilios pumonares, igual o menor de $2 \mathrm{~cm}^{12}$. A la fecha, no hay un consenso internacional sobre la forma de determinar el tamaño del neumotórax. En la población objeto de este informe, se utilizó el índice de Light.

En cuanto al neumotórax oculto, su incidencia varía entre I, $8 \%$ y $26 \%$, con un promedio de $5 \%$, aunque se ha informado hasta una de $64 \%$ en politraumatismos graves ${ }^{2,13,14}$. Se ha intentado identificar factores de riesgo predictores de neumotórax oculto en pacientes con trauma de tórax y trauma grave. Se encontró asociación significativa con enfisema subcutáneo en $22 \%$ de los casos. Todos los pacientes con enfisema tuvieron un neumotórax asociado, abierto u oculto, y no se encontró que estos predijeran otras condiciones, como fracturas costales o contusiones pulmonares ${ }^{3}$.

Se ha intentado mejorar el diagnóstico con estudios adicionales a la radiografía de tórax. La TC y la ecografía han aumentado 3,5 veces los hallazgos torácicos de significado clínico inespecífico o insignificante, con una sensibilidad hasta del 98,1\%. Sin embargo, evidenciar un neumotórax oculto no ha generado cambios significativos en el manejo de muchos de estos casos, diferente a lo que pudiera ser un neumotórax de hasta el 30 $\%$ en pacientes asintomáticos ${ }^{13,15-17}$. En este estudio, se emplearon TC solo en $2,3 \%$ de los casos y ecografía subxifoidea en $27,3 \%$, para evaluar lesiones cardíacas asociadas, no en búsqueda de un neumotórax oculto.

Actualmente, no hay consenso sobre cuándo se debe intervenir ante un neumotórax oculto. Desde hace varios años, el Advanced Trauma Life Support (ATLS) recomienda el tubo de tórax para tratar el neumotórax ${ }^{18}$. Sin embargo, el tratamiento conservador para estos pacientes ha sido una opción que, aunque no se recomienda plenamente por el gran riesgo de desarrollar un neumotórax a tensión, se ha venido practicando a lo largo del tiempo debido a que la toracostomía puede ser mórbida y causar complicaciones hasta en 22 a $30 \%$ de los $\operatorname{casos}^{19,20}$. En varios es- 
tudios y reportes de caso, se ha demostrado que el tratamiento conservador puede ser efectivo y se pueden evitar las complicaciones de la toracostomía ${ }^{1,21,22}$.

En varios estudios se ha encontrado que, cuando la radiografía inicial es negativa o normal en casos de heridas por arma cortopunzante, el neumotórax tardío es infrecuente $(2,2 \%)$ y se requiere la toracostomía en el I,I \% de los pacientes 3,4. Las series anteriores sobre heridas penetrantes de tórax informaban neumotórax o hemotórax tardío en 8 a I2 \% de los casos, y recomendaban vigilancia hasta por 72 horas ${ }^{8,23}$, lo cual no sería práctico en nuestro medio y, posiblemente, no generaría un impacto significativo adicional.

No hay consenso sobre cuándo está indicado el tratamiento conservador según el tamaño del neumotórax. En algunos estudios se sugiere que puede ser efectivo y seguro cuando el neumotórax es del Io al I5 \% o menos de $2,5 \mathrm{~cm}$ del espacio interpleural en la radiografía de tórax, y recomiendan la observación por 24 horas, como mínimo ${ }^{24}$. Se ha calculado una tasa de reabsorción del neumotórax de I, $25 \%$ por día, aproximadamente ${ }^{18,25-27}$. Sin embargo, existen reportes de casos con neumotórax del $30 \%$, tratados de forma conservadora sin complicaciones y con resolución completa ${ }^{19}$. Por la experiencia en este tipo de casos, en el Hospital San Vicente Fundación, los pacientes asintomáticos con neumotórax aislado menor del $30 \%$ reciben tratamiento conservador.

Los datos apuntan a que el manejo conservador puede ser efectivo en pacientes sin neumotórax inicial y con neumotórax menor del $30 \%$. En este caso, el 7I,4\% de los pacientes sin neumotórax inicial y el 3,9\% de aquellos con neumotórax menor del $30 \%$, son dados de alta sin un periodo de observación y sin que reingresen; se debe tener en cuenta que, entre los primeros, se pueden incluir los que presentan heridas no penetrantes. Por otro lado, se dejaron bajo observación I $8,9 \%$ de los pacientes sin neumotórax y $68,9 \%$ de aquellos con neumotórax menor del $30 \%$; el I3,4\% de los primeros y el 29,6 \% de los segundos requirieron toracostomía cerrada como tratamiento definitivo, ya fuera por cambios en los hallazgos radiológicos, por aparición de síntomas o por ambos.

Durante este estudio, más de la mitad (68\%) de los pacientes con neumotórax menor del 30\% en la radiografía inicial se dejaron bajo observación y, en el $73 \%$, el control demostró mejoría o ausencia de cambios con respecto a la radiografía previa. En el $27 \%$ de los pacientes, el neumotórax aumentó o se asoció con hemotórax, y se requirió toracotomía en uno y toracostomía cerrada en 42 (29,6 \%), lo cual correspondió al $32 \%$ de las radiografías de control entre 3 y 6 horas postrauma, $y$ al $25 \%$ de las tomadas después de 6 horas.

En el presente estudio, se tomó un control después de 3 a 6 horas de la radiografía inicial normal o con neumotórax menor del $30 \%$, ambos hallazgos considerados como criterios para un manejo expectante. En 22,2 \% de estos casos (80/36I), se necesitó tratamiento quirúrgico después de la radiografía de control. Gran parte (56 $\%)$ de los estudios de control se tomaron entre las 3 y 6 horas iniciales; no obstante, en $15,7 \%$ de los pacientes que tuvieron que ser intervenidos, fue después de 6 horas del ingreso, lo cual se explica por la gran congestión que presenta el servicio de urgencias del hospital.

En un estudio descriptivo y prospectivo se afirma que, de 87 pacientes bajo observación, solo uno tuvo que ser intervenido a las 3 horas $y$, a las 6 horas, no hubo cambios en el resto de las imágenes y no se necesitaron más intervenciones ${ }^{7}$. En otro estudio se hace referencia a la baja incidencia de neumotórax tardío cuando la radiografía inicial es normal, y a la casi nula posibilidad de que se desarrolle un neumotórax después de 3 horas ${ }^{28}$.

Se han evaluado intervalos de seguimiento radiográficos cada vez más cortos para la exclusión de lesiones y un egreso seguro. Weigelt, et al., en un estudio prospectivo de pacientes asintomáticos con puñaladas torácicas, encontraron que el $9 \%$ desarrolló hemotórax o neumotórax tardío ${ }^{6,29}$. En pacientes seleccionados, se sugiere el seguimiento con una única radiografía a las tres horas del trauma, con lo cual se logra estancias 
hospitalarias más cortas y menor exposición a la radiación.

Otras lesiones torácicas como el hemoneumotórax, requieren tratamiento quirúrgico debido al riesgo de hemotórax coagulado, de empiema o de que no se logre su resolución. En el presente estudio, de los 199 pacientes que presentaron hemoneumotórax, en I88 se practicaron procedimientos quirúrgicos, con un total de 179 (90 \%) toracostomías cerradas. En $20 \%$ de los casos, el neumotórax puede estar asociado con hemotórax ${ }^{30}$. No obstante, los pacientes asintomáticos, con un neumotórax menor del $30 \%$, con neumotórax oculto o con hemotórax menor de $300 \mathrm{ml}$, se podrían manejar de forma expectante ${ }^{31}$.

En este estudio no se logró evaluar el costo económico del periodo de observación y la radiografía de control necesaria para generar un potencial cambio en la conducta médica.

La principal limitación del presente estudio es que, al ser retrospectivo, no se cuenta con el seguimiento de los $722(46,5 \%)$ pacientes dados de alta después de una única radiografía inicial, los cuales no presentaron reingresos o complicaciones en esta misma institución. Ante la ausencia de un sistema de historia clínica unificado y la falta de acceso a la salud de esta población de bajos recursos, no se puede asegurar que no hayan requerido alguna intervención adicional en otra institución. No obstante, se puede considerar que estos pacientes, al igual que aquellos bajo observación cuya radiografía no presentó ningún cambio, pudieran no haberla necesitado.

Teniendo en cuenta que un porcentaje significativo de pacientes que fueron dejados bajo observación necesitaron finalmente de toracostomía cerrada, no se pretende cambiar esta conducta en el servicio de urgencias con base en el presente estudio. Se plantea la necesidad de llevar a cabo estudios prospectivos que permitan determinar los posibles resultados, el tiempo de observación indicado y el potencial beneficio que puedan generar otros exámenes adicionales de bajo riesgo, como la ecografía pleural.

\section{Conclusión}

La toracostomía cerrada en pacientes con neumotórax traumático pequeño o moderado, en ausencia de otras lesiones significativas o necesidad de presión positiva, podría ser innecesaria, al igual que los períodos prolongados de observación y las radiografías de control en pacientes con una radiografía inicial normal o con hallazgos mínimos. Estos pacientes podrían ser dados de alta de forma segura, disminuyendo así la estancia hospitalaria, la irradiación y los costos asociados con la observación.

\section{Cumplimiento de normas éticas}

Declaración de conflicto de intereses: Los autores declaran que no tienen conflicto de intereses.

Consentimiento informado: Este estudio es una revisión retrospectiva de historias clínicas, considerado un estudio sin riesgo según la Resolución 008430 de 1993 del Ministerio de Salud de Colombia; como tal, no requiere de un consentimiento informado. El Comité de Ética Institucional aprobó el diseño y la metodología del estudio.

Fuentes de financiación: Recursos propios de los autores.

\section{Referencias}

I. Mowery NT, Gunter OL, Collier BR, Diaz JJ, Haut E, Hildreth A, et al. Practice management guidelines for management of hemothorax and occult pneumothorax. J Trauma. 2011;70:510-8.

2. Ball CG, Dente CJ, Kirkpatrick AW, Shah AD, Rajani RR, Wyrzykowski AD, et al. Occult pneumothoraces in patients with penetrating trauma: Does mechanism matter? Can J Surg. 2010;53:25I-5.

3. Ball CG, Ranson K, Dente CJ, Feliciano DV, Laupland $\mathrm{KB}$, Dyer D, et al. Clinical predictors of occult pneumothoraces in severely injured blunt polytrauma patients: A prospective observational study. Injury. 2009;40:44-7.

4. Zehtabchi S, Morley EJ, Sajed D, Greenberg O, Sinert R. Delayed pneumothorax after stab wound to thorax and upper abdomen: Truth or myth? Injury. 2009;40:40-3.

5. Ball CG, Kirkpatrick AW, Laupland KB, Fox DL, Litvinchuk S, Dyer DM, et al. Factors related to the failure of radiographic recognition of occult posttraumatic pneumothoraces. Am J Surg. 2005;I89:54I-6; discussion 6. 
6. Berg RJ, Inaba K, Recinos G, Barmparas G, Teixeira PG, Georgiou C, et al. Prospective evaluation of early follow-up chest radiography after penetrating thoracic injury. World J Surg. 2013;37:1286-90.

7. Shatz DV, de la Pedraja J, Erbella J, Hameed M, Vail SJ. Efficacy of follow-up evaluation in penetrating thoracic injuries: 3- vs. 6-hour radiographs of the chest. J Emerg Med. 200I;20:28I-4.

8. Kerr TM, Sood R, Buckman RF, Gelman J, Grosh J. Prospective trial of the six hour rule in stab wounds of the chest. Surg Gynecol Obstet. 1989;169:223-5.

9. Rhea JT, DeLuca SA, Greene RE. Determining the size of pneumothorax in the upright patient. Radiology. I982;I44:733-6.

Io. Collins CD, López A, Mathie A, Wood V, Jackson JE, Roddie ME. Quantification of pneumothorax size on chest radiographs using interpleural distances: Regression analysis based on volume measurements from helical CT. AJR Am J Roentgenol. 1995;165:II27-30.

II. Baumann MH, Strange C, Heffner JE, Light R, Kirby TJ, Klein J, et al. Management of spontaneous pneumothorax: An American College of Chest Physicians Delphi consensus statement. Chest. 2001;II9:590-602.

I2. MacDuff A, Arnold A, Harvey J, Group BPDG. Management of spontaneous pneumothorax: British Thoracic Society Pleural Disease Guideline 20I0. Thorax. 20I0;65(Suppl.2):iir8-3I.

I3. Ball CG, Kirkpatrick AW, Feliciano DV. The occult pneumothorax: What have we learned? Can J Surg. 2009;52:Ei73-9.

I4. Charbit J, Millet I, Maury C, Conte B, Roustan JP, Taourel $\mathrm{P}$, et al. Prevalence of large and occult pneumothoraces in patients with severe blunt trauma upon hospital admission: Experience of 526 cases in a French level I trauma center. Am J Emerg Med. 2015;33:796-80I.

I5. Mollberg NM, Wise SR, De Hoyos AL, Lin FJ, Merlotti G, Massad MG. Chest computed tomography for penetrating thoracic trauma after normal screening chest roentgenogram. Ann Thorac Surg. 2012;93:1830-5.

I6. Kea B, Gamarallage R, Vairamuthu H, Fortman J, Lunney K, Hendey GW, et al. What is the clinical significance of chest CT when the chest X-ray result is normal in patients with blunt trauma? Am J Emerg Med. 2013;31:1268-73.

I7. Blaivas M, Lyon M, Duggal S. A prospective comparison of supine chest radiography and bedside ultrasound for the diagnosis of traumatic pneumothorax. Acad Emerg Med. 2005;12:844-9.
I8. Johnson G. Traumatic pneumothorax: Is a chest drain always necessary? J Accid Emerg Med. 1996;13:173-4.

19. Llaquet Bayo H, Montmany Vioque S, Rebasa P, Navarro Soto S. Resultados del tratamiento conservador en pacientes con neumotórax oculto. Cir Esp. 2016;94:232-6.

20. Tapias L, Tapias-Vargas LF, Tapias-Vargas L. Complicaciones de los tubos de tórax. Rev Colomb Cir. 2009;24:46-55.

2I. Idris BM, Hefny AF. Large pneumothorax in blunt chest trauma: Is a chest drain always necessary in stable patients? A case report. Int J Surg Case Rep. 2016;24:88-90.

22. Kirkpatrick AW, Stephens MV, Fabian T; Evidence Based Reviews in Surgery Group. Canadian Association of General Surgeons and American College of Surgeons Evidence Based Reviews in Surgery. I8. Treatment of occult pneumothorax from blunt trauma. Can J Surg. 2006;49:358-6I.

23. McLatchie GR, Campbell C, Hutchison JS. Pneumothorax of late onset after chest stabbings. Injury. 1980;II:33I-5.

24. Molnar TF. Thoracic trauma: Which chest tube when and where? Thorac Surg Clin. 2017;27:13-23.

25. Kircher LT, Swartzel RL. Spontaneous pneumothorax and its treatment. J Am Med Assoc. I954;155:24-9.

26. Symington L, McGugan E. Towards evidence based emergency medicine: Best BETs from the Manchester Royal Infirmary. Bet I: Is a chest drain necessary in stable patients with traumatic pneumothorax? Emerg Med J. 2008;25:439-40.

27 Henry M, Arnold T, Harvey J, Pleural Diseases Group SoCC, British Thoracic Society. BTS guidelines for the management of spontaneous pneumothorax. Thorax. 2003;58(Suppl.2):ii39-52.

28. Seamon MJ, Medina CR, Pieri PG, Fisher CA, Gaughan JP, Bradley KM, et al. Follow-up after asymptomatic penetrating thoracic injury: 3 hours is enough. J Trauma. 2008;65:549-53.

29. Weigelt JA, Aurbakken CM, Meier DE, Thal ER. Management of asymptomatic patients following stab wounds to the chest. J Trauma. 1982;22:29I-4.

30. Mabry R, McManus JG. Prehospital advances in the management of severe penetrating trauma. Crit Care Med. 2008;36(Suppl.):S258-66.

3I. Neff MA, Monk JS, Peters K, Nikhilesh A. Detection of occult pneumothoraces on abdominal computed tomographic scans in trauma patients. J Trauma. 2000;49:28I-5. 